\title{
Knowledge Exploratory for Service Management and Innovation
}

\author{
Yoshinori Hara \\ Graduate School of Management, Kyoto University \\ Yoshida-Honmachi, Sakyo-ku, Kyoto 606-8501, Japan \\ haralgsm.kyoto-u.ac.jp
}

\begin{abstract}
In this talk, various trends in the current service economy are explained. Global economy has turned out to be a service-oriented economy, in addition to the fact that the economy is going along with more digitized information rather than just labor intensive human service activities. In Japan's case, for example, more than $70 \%$ of GDP has been created by service sectors in a broader sense recently, and the ratio is still going up.

However, the problem we are facing to the service-oriented economy emerges due to the uncertainness of service management and the low productivity of service businesses in general. We explain how knowledge exploratory could contribute to providing a solution or an insight to improve the situation as "service innovation", and to discuss framework and/or design process of service innovation, illustrating with some of the actual research and education activities. They include service literacy management, service blueprinting based on knowledge management framework and common data models such as UML. The goal of this talk is to provide a common view of the knowledge management framework that will support service innovation for the service-oriented economy. We believe that this kind of systematic approach, having human beings located in the system, will have more meaningful implications for the new economy.
\end{abstract}

